\title{
Advances in instrumentation at the W. M. Keck Observatory
}

\author{
Sean M. Adkins ${ }^{*}$, Taft Armandroff ${ }^{\mathrm{a}}$, Hilton Lewis ${ }^{\mathrm{a}}$, Chris Martin ${ }^{\mathrm{b}}$, \\ Ian S. McLean ${ }^{\mathrm{c}}$, Constance Rockosi ${ }^{\mathrm{d}}$, Peter Wizinowich ${ }^{\mathrm{a}}$ \\ ${ }^{a}$ W. M. Keck Observatory, 65-1120 Mamalahoa Highway, Kamuela, HI, USA 96743; \\ ${ }^{\mathrm{b}}$ California Institute of Technology, 1200 E. California Blvd., Pasadena, CA 91125, USA \\ ${ }^{\mathrm{c}}$ University of California, Los Angeles, Box 951547, Los Angeles, CA 90095-1547 \\ ${ }^{\mathrm{d}}$ UCO/Lick Observatory, 1156 High St., Santa Cruz, CA, USA 95062
}

\begin{abstract}
In this paper we describe both recently completed instrumentation projects and our current development efforts in the context of the Observatory's science driven strategic plan which seeks to address key questions in observational astronomy for extra-galactic, Galactic, and planetary science with both seeing limited capabilities and high angular resolution adaptive optics capabilities. This paper will review recently completed projects as well as new instruments in development including MOSFIRE, a near IR multi-object spectrograph nearing completion, a new seeing limited integral field spectrograph for the visible wavelength range called the Keck Cosmic Web Imager, and the Keck Next Generation Adaptive Optics facility and its first light science instrument DAVINCI.
\end{abstract}

Keywords: Adaptive Optics, Instrumentation, Infrared, Multi-object, Spectroscopy, Visible

\section{INTRODUCTION}

For the past 15 years the W. M. Keck Observatory (WMKO) has played a leading role in U.S. astronomy and astrophysics. The two $10 \mathrm{~m}$ Keck telescopes, located at one of the world's premier sites for astronomy, were the first of a new generation of very large ground-based optical/infrared telescopes with the first Keck telescope beginning science operations in May of 1993, and the second Keck telescope starting science operations in October of 1996. The telescopes feature a highly capable suite of advanced instrumentation for both optical and near-infrared wavelengths, including imagers, multi-object spectrographs, high-resolution spectrographs, and integral-field spectroscopy. WMKO has developed and operates a sophisticated natural and laser guide star (LGS) adaptive optics (AO) system and related instrumentation. The Observatory also operates the only large-aperture infrared interferometer in the U.S. ${ }^{[1],[2]}$ WMKO is a versatile, efficient, high-impact general-purpose observatory. Its success relies on these closely knit factors: a clear strategic direction, an extraordinary operations staff, a highly-talented and innovative instrument-building community, a creative, enthusiastic, involved, and broad user community, and a suite of efficient, state of the art instruments that keep the observatory at the cutting edge. It is our intention to sustain the Observatory as one of the crown jewels of U.S. astronomy in the coming decade.

Although in past papers we have provided a complete retrospective of the instrumentation capabilities at $\mathrm{WMKO}^{[3]}$, in this paper after providing some background information to establish the strategic context for our instrumentation program we will focus on recently completed and current instrumentation developments.

\section{BACKGROUND}

The Observatory was constructed with private funds donated by the W. M. Keck Foundation and is operated as a partnership among the California Institute of Technology (CIT), the University of California (UC), and NASA. The Observatory's headquarters are in Waimea, HI on Hawaii Island with the Observatory facilities located on the summit of Mauna Kea at $4160 \mathrm{~m}$ above sea level. Instrumentation is developed through collaborations between WMKO and academic and commercial organizations. The main centers for the construction of WMKO instrumentation are the optical/infrared group at CIT, the Infrared Laboratory at the University of California, Los Angeles (UCLA), and the UCO/Lick Observatory located on the campus of the University of California, Santa Cruz (UCSC).

*sadkins@keck.hawaii.edu; Phone: (808) 885-7887; Fax: (808) 885-4464; http://www.keckobservatory.org/ 


\subsection{The WMKO Observing Community}

Access to science observing time with the Keck telescopes is determined by time allocation committees (TACs) representing the institutions with direct interests in the Observatory: CIT and UC each with a $35 \%$ share, NASA with a $16.5 \%$ share and the University of Hawaii (UH) with a $12.5 \%$ share. Through participation in the Telescope System Instrumentation Program (TSIP), WMKO has made available 92 nights between 2006 and 2009 to the National Optical Astronomy Observatory (NOAO). In addition, exchanges with the Gemini Observatory over the last 4 years have totaled 29 nights. The NASA, NOAO, and Gemini time are directly available to the entire U.S. community. In a search of the observing database from 2006 to 2009 a total of 873 unique names were found with the following institutional breakdown: $597 \mathrm{UC} / \mathrm{CIT} / Y a l e, 156 \mathrm{UH}, 146$ NASA, $108 \mathrm{NSF}, 22 \mathrm{Gemini}, 24$ Subaru, and 25 WMKO. The recent ALTAIR survey ${ }^{[4]}$ to understand the needs of the U.S. community for observing resources on large telescopes stated “...when asked to prioritize possible avenues by which the large telescope system could by enhanced with any increases in federal funding, the highest priority of respondents was for more open access time on non-federal facilities" (p. 14) and "For example, the access to the additional capabilities on the non-federal facilities that is afforded by TSIP and the NASA open access time on Keck is highly valued by the community" (p. 15), reflecting the demand for Keck telescope access among others.

\subsection{Broader Impacts}

The impact of the Keck telescopes on astronomy education is well illustrated by the $194 \mathrm{PhD}$ theses produced using Keck telescope data through early 2010. Keck AO data has been used in a significant number of these theses, including 7 of the 18 Keck-based theses published in 2009. As another example, refereed science papers published in 2005 based on Keck AO data included 25 graduate students and 20 post doctoral research associates as co-authors. WMKO is currently providing many graduate students and post doctoral research associates direct access to the state-of-the-art LGS AO science facility. The impact of Keck AO and the Keck Interferometer on educating technical people has also been high, with numerous post doctoral research associates ( $\sim 10$ to date for AO, 3 for Interferometry) and young engineers and astronomers involved in development and operations.

\subsection{The WMKO Strategic Plan}

Instrumentation development at the Observatory is guided by a science driven, community based strategic plan. This plan emphasizes observational capabilities that are aligned with the scientific interests of our observing community and covers two major areas, seeing limited spectroscopy in the visible and near-IR wavelengths, and high angular resolution imaging and spectroscopy. The major attributes of the Observatory's seeing limited spectroscopic capabilities are high sensitivity with either a high object multiplex or high spectral resolution. The Observatory's high angular resolution capabilities include the world's most scientifically productive LGS AO system ${ }^{[5]}$ and the only U.S. based large aperture infrared interferometer which employs the two Keck telescopes to create an interferometer with an $85 \mathrm{~m}$ baseline ${ }^{[6]}$.

The WMKO strategic plan articulates four science driven themes that guide technical developments at the Observatory. These are high angular resolution, state of the art instrumentation, highly efficient operations, and flexibility. Through an annual process of review the strategic plan is updated, and potential new projects are considered and mapped to each of the themes. The Observatory then uses this list of candidate projects to guide its annual planning and fund raising activities.

\subsection{The WMKO Development Program}

The Observatory's instrumentation projects span a range of project scales from major new instrumentation developments, to upgrades to existing instruments, and to many smaller projects aimed at improved performance, adding features to enhance usability, or to address issues uncovered during routine operations. Major development projects and upgrades are managed through the Observatory's development programs in instrumentation and optics. Smaller projects are managed by the operations and observing support groups at the Observatory.

The development philosophy at the Observatory is based on a consistent set of project phases and standards aimed at creating an environment and supporting infrastructure that maximizes the success of each project. The Observatory provides program level oversight for each development project and statements of work define the roles and activities of each of the project teams. Project management is an essential part of each project team, and large projects are normally required to have a full time project manager. Observatory staff members are also part of the project team, and often participate in the development of the instrument as well as taking responsibility for the interfacing of the instrument with 
the Keck telescopes. While the Observatory holds full authority over each project we view the collaborative and cooperative side of the relationship as the most important element in ensuring a successful outcome.

\section{NEW DEVELOPMENTS IN INSTRUMENTATION}

\subsection{LRIS Red Channel Detector Upgrade}

LRIS, the low resolution imaging spectrograph ${ }^{[7]}$ is a Cassegrain instrument on the Keck I telescope providing multi-object spectroscopy and imaging using red and blue optimized cameras to cover the visible wavelength range from 310 to $1000 \mathrm{~nm}$. The instrument was commissioned in 1993, and the blue channel was upgraded to a mosaic of two $2 \mathrm{k} \mathrm{x}$ $4 \mathrm{k}$ CCDs from e2v technologies in 2002. In 2005 in collaboration with the technical facilities group of the UCO/Lick Observatory we began the development of an upgrade for the red channel to a mosaic of two $2 \mathrm{k} \times 4 \mathrm{k}$ deep depletion, thick substrate, $\mathrm{CCDs}^{[8]}$ with $15 \mu \mathrm{m}$ pixels from Lawrence Berkeley National Laboratory (LBNL). The co-principal investigators for this upgrade were Michael Bolte and Constance Rockosi from UCO/Lick Observatory. The detailed design review for this upgrade was passed in March 2008 and the full scale development phase was completed with a pre-ship review held in April 2009. Installation on the LRIS instrument took place in May 2009, with first light observations in June $2009^{[9]}$.

The red channel of LRIS had not been upgraded since delivery and was equipped with a SITe $2 \mathrm{k} x$ 2k CCD with $24 \mu \mathrm{m}$ pixels. The read out electronics was an Astronomical Research Cameras (ARC) generation I system. The primary objective of the LRIS red upgrade was to obtain improved sensitivity at the long wavelength end of the red channel. The larger imaging area provided by the new detectors in the spectral direction $(61.44 \mathrm{~mm})$ allows an increase in spectral coverage of $\sim 25 \%$. In the spatial direction the mosaic is $65.23 \mathrm{~mm}$ across including the gap between the two detectors. Because the slit mask limits the field of view (FOV) the coverage increase in the spatial direction for spectroscopy is minimal. For imaging the full area of the mosaic is useable with some vignetting $(\sim 20 \%)$ at the corners of the array. Beyond the benefits of the new CCDs, additional objectives for the upgrade were to increase the hold time of the red side liquid nitrogen dewar to at least 24 hours to match the hold time of the blue side dewar, avoiding any significant increase in flexure in the red channel, and ensuring that the performance of the blue channel was unaffected by the upgrade. In order to minimize unintended effects and control costs the new dewar and electronics had to fit within the space available on the instrument, and the dewar was required to interface to the existing shutter assembly and red channel camera. One important issue for the upgrade was the need to replace the field flattener element of the camera, which mounts on the dewar in from of the vacuum window. The field flattener of the existing red channel has a prescription designed to partially compensate for the curvature of the imaging surface of the SITe CCD.

Focusing the red channel requires the CCD, dewar window and field flattener to translate along the optical axis with respect to the camera. In order to make room for the new dewar the focus stage which held the red side dewar was redesigned to employ a low profile wedge mechanism using linear slides and a motor driven ball screw. The new dewar was constructed using electro-polished CRS for the outer can and an electro-polished copper inner can that is gold plated on the vacuum side. The new dewar was also equipped with an ion pump which maintains a higher vacuum in the dewar, extending the hold time and reducing the frequency of maintenance.

The detectors are mounted in a low profile mount with G-10 thermal standoffs that also serve as flexures. The detector mount is shielded with tantalum and equipped with a focal plane mask near the CCDs to shield the light sensitive output amplifiers and also block light from the aluminum nitride (AlN) substrate on which the CCDs were mounted. AlN is phosphorescent, and we observed that the AlN used to mount the CCDs would emit light for a considerable time when cold if it was exposed to normal room lighting. The assembly consisting of the new dewar, the new focus stage, and the existing shutter and red camera is shown in Figure 1.

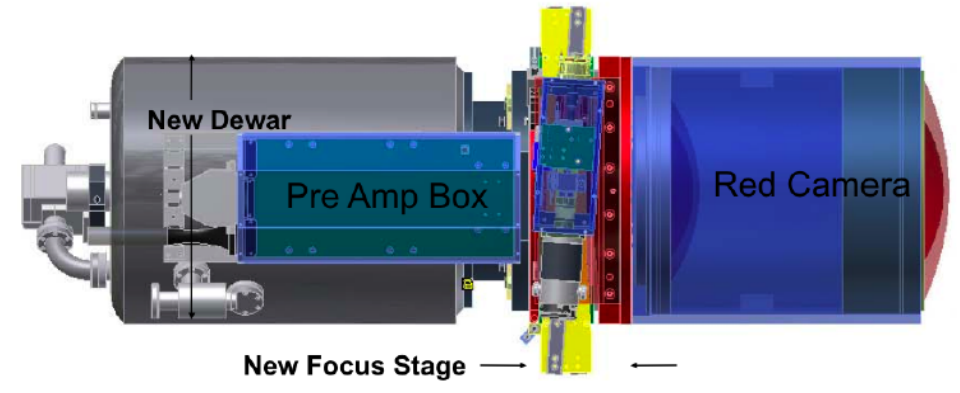

Figure 1: LRIS red channel upgrade dewar and focus stage with the existing red channel camera 
Because of the restricted space available for the dewar and focus stage on LRIS, and the need to remove the dewar and focus stage to service the shutter or to perform work on the dewar itself, the structural (non-moving) elements of the focus stage and a mockup of the new dewar were constructed in the preliminary design phase. The new CCD controller enclosure was also mocked up and these mockups were test fitted on LRIS at the summit. This allowed the Observatory's instrument engineering staff to work with the LRIS upgrade design team to ensure that the removal and replacement procedures were safe and practical. This prototyping effort worked very well and resulted in a high level of satisfaction with the final product from an operations point of view. In the detailed design phase the prototype of the focus stage was completed by adding the moving components and the prototype was then subjected to flexure testing. Based on the results of this testing the design was then refined during the full scale development phase.

Figure 1 also shows a pre-amp box which contains a custom circuit board with four pre-amplifiers for the CCD video outputs and a set of analog switches that isolate the CCDs from the controller so that power-on self testing of the CCD controller can be performed prior to connecting the controller to the CCDs. The pre-amp board also incorporates I2C based voltage monitoring that is used to measure the clock and bias voltages at the dewar, supporting testing and diagnostics without the need to remove the dewar for access to physical test points.

The CCD controller is an Astronomical Research Cameras generation II system and uses a dual channel video board for each CCD along with the standard timing, bias, and utility boards. An additional bias voltage board was required (supplied by LBNL) to generate the positive high voltage (up to $100 \mathrm{Vdc}$ ) substrate bias required by the deep depletion CCDs, and additional negative bias voltages also required for these CCDs. Connections from the controller to the dewar are made using a custom front panel interconnect board that simplifies the cabling and also provides a connection for the shutter controller.

Installation of the upgrade went very smoothly thanks to the early mechanical prototyping, and careful planning and packaging efforts for the support equipment (power supplies, ion pump controller, and shutter controller) that had to be added to the LRIS red channel electronics bay. Commissioning results showed that the sensitivity of the new CCDs was excellent, achieving the goal of enhanced red sensitivity and providing the anticipated additional spectral coverage. The sensitivity gain was measured at $\sim 1.4$ times in the I band, and after correction for the atmosphere the total throughput, including the telescope is $30 \%$ at $900 \mathrm{~nm}$, and remains above $20 \%$ at $950 \mathrm{~nm}$. The dark current is very low due to the operating temperature of $-140{ }^{\circ} \mathrm{C}$, with the maximum exposures limited to approximately 20 minutes primarily by the sensitivity of the CCDs to radiation events ("cosmic rays"). The read noise is also excellent at 3.1 to 4.7 electrons per read across the four output amplifiers. The upgrade also achieved the objective of increased red side hold time with $\sim 28$ hours maximum between fills, and the performance of the blue channel was unaffected by the red channel upgrade. The goal of not increasing the red side flexure was not achieved; flexure on the red side was increased by $\sim 30 \%$ in the spatial direction. However, while the thin substrate of the original SITe CCD resulted in significant fringing at the longer wavelengths, a particular problem for LRIS due to the flexure in the instrument, with the new CCDs the amplitude of the fringing at the longest wavelengths is below the residual variations after flat fielding.

\subsection{MAGIQ Guider Upgrades}

In 2008 the Observatory commissioned the first acquisition and guide camera hardware upgrade on the NIRSPEC instrument $^{[10]}$ as part of its program called MAGIQ, the Multi-function Acquisition, Guiding and Image Quality monitoring system, to upgrade all of the acquisition and guiding hardware and software at the Observatory. In 2009 we upgraded the slit viewing camera on LRIS, and replaced the LRIS movable offset guider with a fixed offset guider. The upgrades used our standard MAGIQ camera, a customized version of the Little Joe camera from SciMeasure Analytical Systems equipped with an e2v technologies CCD47-20BT. The upgrade to the slit guider provided improved sensitivity, but was otherwise largely unchanged with the exception of replacing the rarely used photometric filters with a set of neutral density filters, and providing a remote focus control. Although the new detector offered a larger FOV, vignetting by other parts of the instrument at the slit plane and limited options for placement of the camera prevented the upgrade from increasing the area of the slit plane that can be viewed with the new camera. The upgrade to the offset guider provides a significant increase in the instantaneous FOV, from 1.36' x 1.02' with the original guider to $2.97^{\prime}$ x $2.97^{\prime}$ with the new offset guider. Note that the original guider was moveable, allowing patrol of a 6 ' swath perpendicular to the 1.36' dimension, but the focus compensation needed as this off-axis guider was moved over the curved focal surface of the telescope was imperfectly achieved and the movable stages used in the guider optical train were a source of maintenance concerns. 
The new offset guider is located closer to the telescope optical axis which is important to support our wavefront sensing method used to measure and control the focus of the telescope. This method uses a deployable prismatic element that divides the light from a reference star into a pattern of six spots on a radius of $\sim 5 "$. Using a modal wavefront estimation technique we are able to determine Zernike coefficients up to Z7 and Z8 (coma) allowing the measurement and correction of telescope focus. We are also able to sense and correct coma introduced by tip-tilt of the telescope secondary. This correction is of particular importance to the LRIS guider implementation since tip-tilt of the secondary displaces the curved focal surface of the telescope. The LRIS science field and the offset guider are both located off the axis of the telescope, and if the telescope is not properly collimated the result would be a focus offset between the guider and the LRIS science field. The new guider also provides a set of photometric filters (B, V, R, and I), four neutral density filters (ND1 through 4), and remote focusing.

The MAGIQ guide cameras are controlled by the MAGIQ software ${ }^{[11]}$ which is also designed to allow control of the existing or "legacy" guide cameras at the Observatory. While we would have preferred to upgrade all of the instrument's camera hardware as quickly as possible, making upgrades to working instruments is difficult to schedule and requires careful attention to the risks imposed on the instrument's availability to science. By allowing operation of the existing guide cameras with the MAGIQ software we gained the numerous operational benefits provided by the new software, and also made the transition to using the new software easier for our support astronomers and observing assistants. Over a 9 month period after the initial release on NIRSPEC we made the transition to the MAGIQ software for the majority of our instruments and for target acquisition with $\mathrm{AO}$ and interferometry.

Since commissioning the LRIS offset guider in the fall of 2009 we have been using the offset guider's wavefront sensing capability to measure the telescope focus and secondary tip-tilt both before and after focus adjustments made using our Mira softwar ${ }^{[12]}$. By comparing the measurements made using the new wavefront sensing technique to the results obtained with Mira we have acquired a statistical database that allows us to evaluate the performance of the technique and establish its readiness as a replacement for Mira, an achievement that will result in saving 15 to 30 minutes of observing time on each LRIS night. Figure 2 shows the results of our measurements to date. The left side of Figure 2 shows the correlation between Mira and MAGIQ focus measurements, the red box indicates the limit of the acceptable focus error. The right side of Figure 2 shows the calibration of measured coma with respect to induced secondary tilt. Our results indicate that performance of the method is good, and after some additional engineering tests to evaluate the effect of telescope elevation and instrument rotator angle on the performance of the measurements we plan to release the MAGIQ telescope focus control for routine use with LRIS.
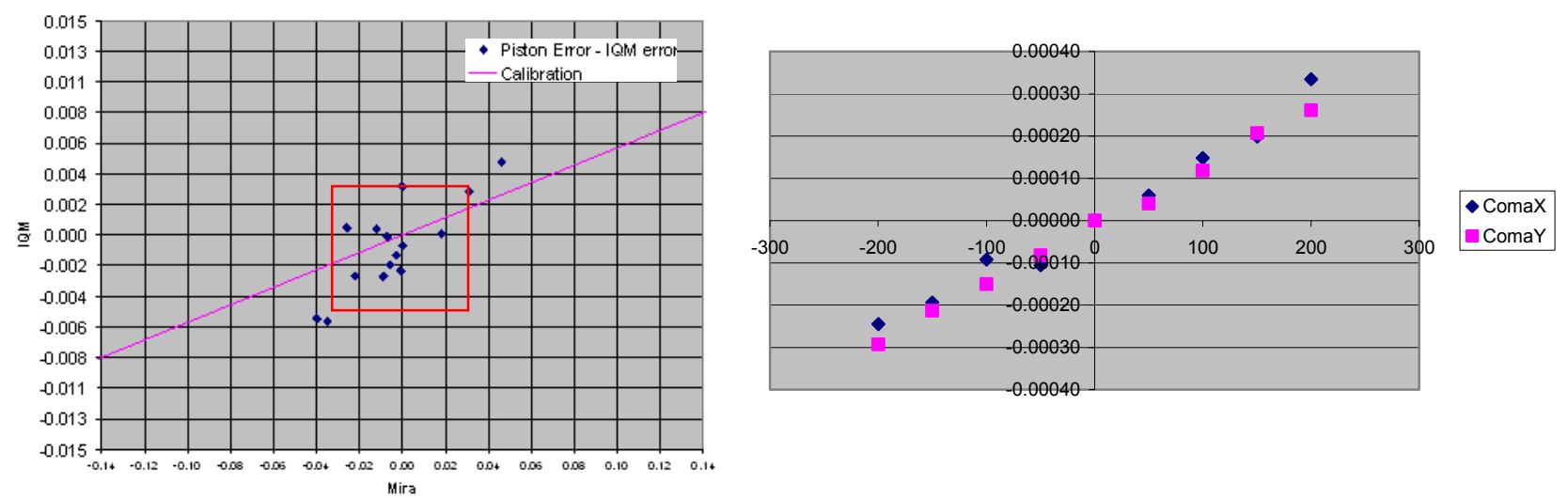

Figure 2: Left: Keck I telescope secondary piston error (in $\mathrm{mm}$ ) measured by the LRIS offset guider wavefront sensing method (IQM) vs. Mira. Right: measured coma (in degrees) vs. Keck I secondary tilt in arc seconds

\subsection{Keck I Laser and LGS Facility}

In September 2005 WMKO and the Gemini Observatory began a collaboration to develop $589 \mathrm{~nm}$ wavelength guide star lasers for the Keck I telescope and the Gemini South telescope with co-technical managers from WMKO (Sean Adkins) and Gemini (Céline d'Orgeville). The laser development was carried out by Lockheed Martin Coherent Technologies (LMCT) under contract with the Association of Universities for Research in Astronomy (AURA). The requirements for the Keck I laser were for a minimum of 20 watts of optical power at $589 \mathrm{~nm}$, and the minimum power requirement for the Gemini South laser was 50 watts at $589 \mathrm{~nm}$. Both lasers were required to have an $\mathrm{M}^{2}<1.4$, a bandwidth $<1.5 \mathrm{GHz}$, 
and a linear output polarization with a purity of $95 \%$ or better. The lasers were required to provide wavelength tuning to allow matching the laser wavelength to the D2a line of the mesospheric sodium layer, and to allow tuning off of resonance with the sodium layer to permit measurement of the background due to Rayleigh scattering of the beam when it is projected upwards to the sodium layer. In the case of the Gemini South LGS AO system the 50 watt output of the laser would be divided into 5 beams, potentially increasing the impact of the overlap between the LGS images on the sodium layer and the Rayleigh background from the 5 beams. The specifications called for a minimum off line tuning range of $5 \mathrm{GHz}$ and a frequency stability of $\leq 100 \mathrm{MHz}$. The lasers were expected to be fully remote controlled and to provide built in beam diagnostics and other measurement facilities to allow efficient routine operation at each observatory.

The design developed by $\mathrm{LMCT}^{[13]}$ uses two high power infrared sources based on mode locked oscillators followed by one or more stages of power amplification. One source operates at $1064 \mathrm{~nm}$, and the second source operates at $1319 \mathrm{~nm}$. The two sources are combined in a lithium triborate (LBO) crystal in a single pass configuration. The crystal is heated to provide non-critical phase matching for the non-linear conversion process of sum frequency generation used to produce the $589 \mathrm{~nm}$ output wavelength. Frequency tuning of the laser is accomplished by a piezoelectric actuator adjusting the angle of an etalon in the $1064 \mathrm{~nm}$ source. A phase modulation spectrometer monitoring a sample of the laser output beam passing through a sodium gas cell is used to determine the output wavelength of the laser. A digital servo loop implemented in the laser's control computer compares the signal from the spectrometer to a desired frequency set point and controls the tuning etalon in the $1064 \mathrm{~nm}$ source to stabilize the output frequency of the laser. This technique has resulted in excellent frequency stability, typically $<20 \mathrm{MHz}$ for the Keck I laser. The Keck I laser exceeded its output power requirement by $50 \%$ and reliably generates $30 \mathrm{~W}$ of power at $589 \mathrm{~nm}$ with an $\mathrm{M}^{2}<1.2$. The bandwidth of the delivered laser was wider than specified at $\sim 1.8 \mathrm{GHz}$. It proved impractical to reduce this bandwidth while maintaining adequate stability of the mode locked oscillators used in the infrared sources. The Keck I laser was delivered to WMKO in September 2009 and installed at the summit in December 2009.

The Keck I laser is installed in a temperature controlled clean room enclosure on the Keck right Nasmyth platform. The laser is projected onto the sky by a $50 \mathrm{~cm}$ aperture launch telescope constructed by Galileo Avionica and mounted behind the Keck I secondary mirror ${ }^{[14]}$. The baseline plan for transporting the beam from the laser to the launch telescope was to use a solid core photonic crystal fiber with a $1 / \mathrm{e}^{2}$ mode field diameter $>10 \mu \mathrm{m}$. A beam injection module located inside the laser enclosure couples the laser output to the fiber, and a second module located at the launch telescope couples the fiber output to the launch telescope. The injection module includes servo motor driven tip-tilt mirrors and position sensing photodiodes to allow remote alignment of the laser output to the injection module optical path. This also provides the ability to compensate for any pointing variations at the laser output. The module at the launch telescope incorporates a shutter and beam dump, a power monitor, and remotely adjustable mirrors to align the beam to the launch telescope. A camera views the beam after transport by the fiber via a low reflectivity mirror to permit monitoring the beam quality.

The laser was first propagated from the Keck I telescope in January 2010. Figure 3 shows the laser propagating from the launch telescope mounted behind the telescope secondary. The full moon illuminates the dome and telescope structure. The results of on sky testing show that the launch telescope produces good beam quality, and all of the control and supervisory systems were demonstrated and perform as designed. Unfortunately the narrow output pulses of the laser and the correspondingly high peak power leads to self phase modulation (SPM) in the transport fiber due to the Kerr effect. As a consequence less than 1 watt of power can be delivered to the fiber before SPM broadens the spectral profile of the laser well beyond the D2a and D2b lines. The approach of using fiber transport has now been abandoned and a free space beam transport system is being designed. Installation of this system is planned for late 2010, followed by further testing. The OSIRIS science instrument will then be relocated from the Keck II telescope to Keck I and commissioned for science operations with the Keck I AO system

Since the Observatory began offering LGS AO for science observing on the Keck II telescope in 2004 the demand for LGS AO has steadily increased. We look forward to commencing LGS AO science operations on the Keck I telescope. Once LGS AO operations begin on Keck I the Observatory will offer LGS AO observing on both telescopes, and LGS $\mathrm{AO}$ correction will also be available for use with the Keck Interferometer. 


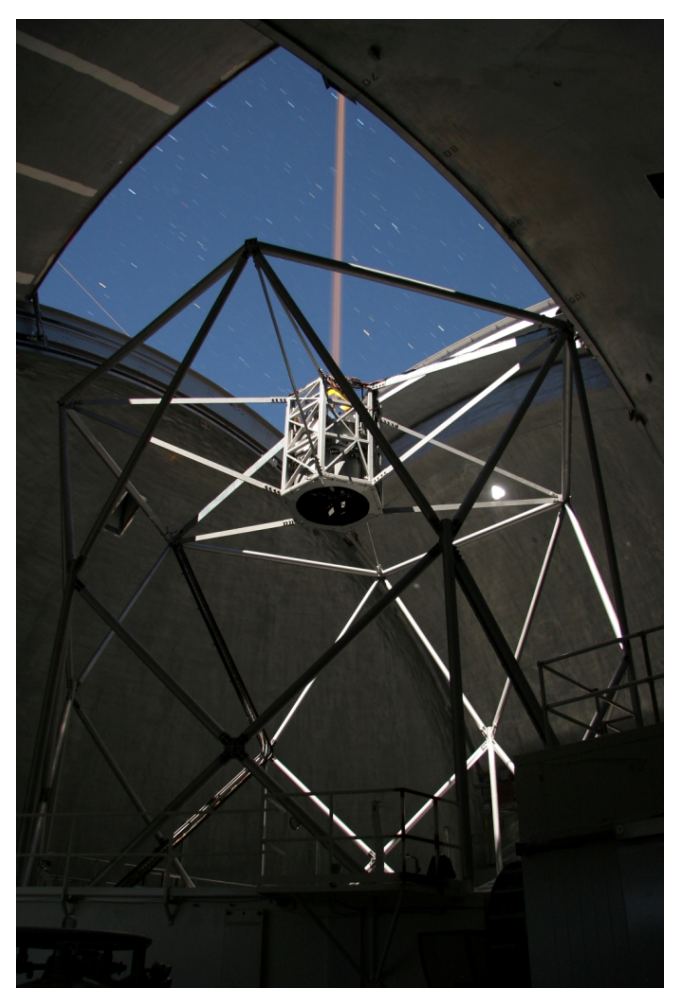

Figure 3: First propagation of the Keck I laser on sky, January 2010

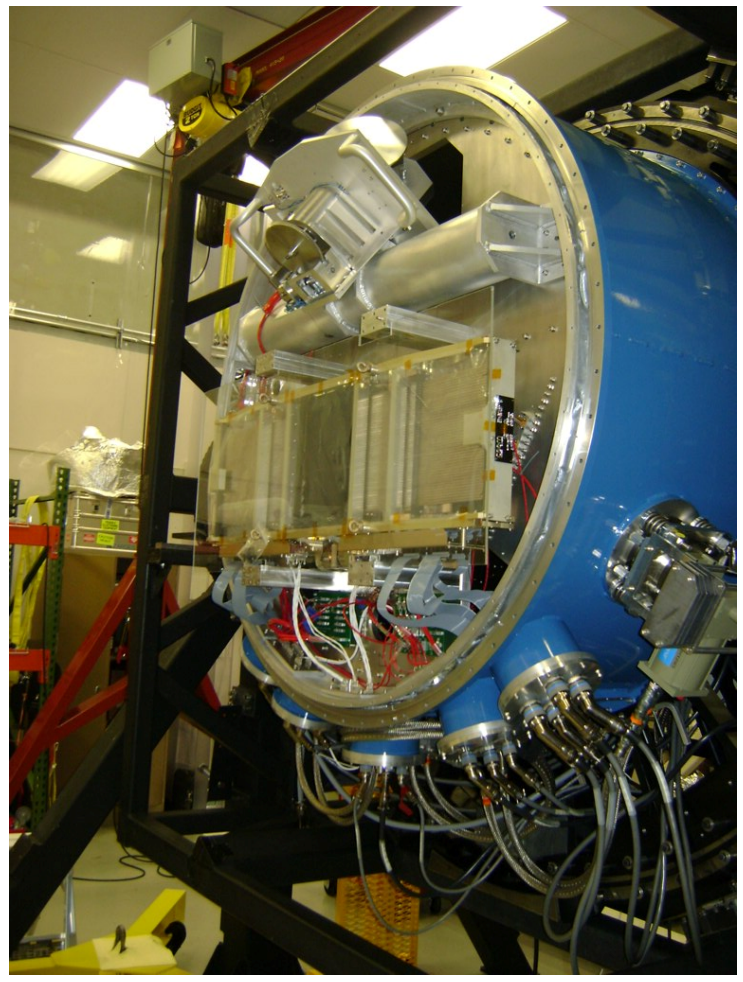

Figure 4: Front view of MOSFIRE during assembly with the front cap removed

\subsection{MOSFIRE}

MOSFIRE is a near-IR ( $\sim 0.97$ to $2.45 \mu \mathrm{m})$ spectrograph for the Cassegrain focus of Keck $\mathrm{I}^{[15]}$. Development began in 2005, and MOSFIRE passed its detailed design review in April of 2007. Design and construction of the instrument is being done in collaboration with CIT, the Infrared Laboratory at UCLA, and UCSC. The principle investigator for MOSFIRE is Ian McLean (UCLA).

MOSFIRE's optical design provides imaging and multi-object spectroscopy over a FOV of 6.14' x 6.14' with a resolving power of $\mathrm{R} \sim 3,270$ for a slit width of 0.7 " (2.9 pixels in the dispersion direction). The detector is a Teledyne Imaging Sensors $2 \mathrm{k} \times 2 \mathrm{k}$ Hawaii $2 \mathrm{RG} \mathrm{HgCdTe}$ array with a long wavelength cut-off of $2.5 \mu \mathrm{m}$. A special feature of MOSFIRE is that its multiplex advantage of up to 46 slits is achieved using a cryogenic configurable slit unit or CSU (developed in collaboration with the Swiss Centre for Electronics and Micro Technology, CSEM) that is reconfigurable under remote control in less than 5 minutes without any thermal cycling of the instrument. Slits are formed by moving opposable bars from both sides of the focal plane. An individual slit has a length of 7.1" but bar positions can be aligned to make longer slits. When the bars are removed to their full extent and the grating is changed to a mirror, MOSFIRE becomes a widefield imager.

The optical design is refractive. A large (388 mm full diameter) entrance window is required to accommodate the $6.8^{\prime}$ diameter field of view. This window is located in a tube or snout at the front of the dewar. To prevent condensation from forming as the center of this large window cools by radiation, a second, thinner (17 mm) Infrasil-302 window is located $228.6 \mathrm{~mm}$ away in the vacuum. An aluminum tube and baffles between the windows are resistively heated to provide radiation to maintain the outer window above the average Observatory dome temperature. The 6 element collimator contains a large $\mathrm{CaF}_{2}$ field lens separated from a second lens group. A folding flat placed between the field lens and the second lens group is equipped with piezo actuators to provide tip-tilt for flexure compensation at the 0.1 pixel level. The (monochromatic) pupil diameter is $125 \mathrm{~mm}$. A pupil mask mechanism provides a rotating pupil mask matched to the shape of the Keck telescopes' segmented primary mirror. The matched mask is moved out of the beam at one end of the rotation travel to allow an oversized circular mask for the $\mathrm{Y}$ and $\mathrm{J}$ bands. A turret mechanism is located after the pupil 
mask. The turret mechanism carries a plane mirror for imaging mounted back to back with the spectrograph grating. Two fixed positions for the grating are provided and order-sorting filters give essentially full coverage of the K, H, J, and $\mathrm{Y}$ bands using the $3 \mathrm{rd}$, 4th, 5 th or 6 th order respectively. The camera has a $250 \mathrm{~mm}$ focal length and a $270 \mathrm{~mm}$ entrance aperture such that it operates at $\mathrm{f} / 0.93$ (under-filled), which is unprecedented for a cryogenic infrared camera of these dimensions and covering this wavelength range. The H-2RG detector is mounted in a detector head assembly with a thermal link to a 2 stage closed cycle refrigerator (CCR) and equipped with a motor driven titanium flexure for focus adjustment to permit optimization of the focus when the instrument is cold.

The MOSFIRE dewar has tori-spherical vacuum end-caps at both ends. Each end cap is removable for servicing, and the intent of the design is to make all of the mechanisms accessible for servicing after removal of either the front or the rear end cap. The vacuum shell must safely resist the atmospheric pressure load in combination with the loads of external components and also have sufficient stiffness to keep the relative motion between internal components within acceptable limits. To meet these requirements, a tubular vacuum shell was chosen based on its efficiency under vacuum loads combined with its uniform stiffness under radial bending loads. Because MOSFIRE is a Cassegrain instrument, it will experience a varying gravity vector during operation, and to minimize flexure all of MOSFIRE's internal components are mounted on two optical benches connected by an inner and outer tubular support structure. Figure 4 shows the front end of MOSFIRE with the front cap removed and showing the CSU at the front center and the grating turret above it attached to a large horizontal support tube.

The optical benches and all internal components except for the detector are cooled to $120 \mathrm{~K}$ by two variable speed single stage CCRs. A closed loop temperature controller with resistive heaters stabilizes the internal temperature to within $1 \mathrm{~K}$ to ensure consistent optical performance. The tubular internal structure efficiently fits the cylindrical envelope of the Cassegrain location on the telescope and accommodates the internal structure. The design is such that both the internal structural support tube and the external cryostat barrel have one common end attached to an instrument mounting "ring" structure that is mounted in a rotator module equipped with a drive and encoder system to provide field derotation during observations.

As of this writing MOSFIRE is in cool down 5 with all of the optics, mechanisms, and the science detector installed. First light in the lab, including arc line spectra as well as imaging is expected in early July 2010. Delivery of MOSFIRE to the Keck I telescope is expected before the end of calendar 2010.

\subsection{The Keck Cosmic Web Imager}

The Keck Cosmic Web Imager (KCWI) is a wide field, seeing limited, integral field spectrograph (IFS) optimized for precision sky limited spectroscopy of low surface brightness phenomena ${ }^{[16]}$. KCWI will feature high throughput and flexibility in field of view (FOV), spatial sampling, bandpass, and spectral resolution. KCWI will provide full wavelength coverage $(0.35$ to $1.05 \mu \mathrm{m})$ using optimized blue and red channels. The preliminary design phase of KCWI has recently been initiated in collaboration with CIT and UCSC. The principle investigator for KCWI is Chris Martin (CIT).

KCWI is based on the Cosmic Web Imager (CWI) recently commissioned at the Palomar Observatory. CWI is a single channel instrument with a fixed spatial sampling scale and a single adjustable VPH grating and an articulated camera with $2 \mathrm{k} \times 4 \mathrm{k}$ mid-band optimized CCD. KCWI will build on the heritage of CWI with a two channel design offering three selectable spatial sampling scales and a selection of VPH gratings. KCWI will be located at the right Nasmyth focus of the Keck II telescope. A plan view of an initial concept for the opto-mechanical layout of KCWI is shown in Figure 5 .

Starting at the left hand side of Figure 5, the light from the telescope passes through the k-mirror image de-rotator and comes to a focus on the image slicer mirrors in the integral field unit (IFU). Light from the slicer mirrors is reflected back to the left and reaches a fixed pupil mirror array which has a center opening to allow the light from the telescope to reach the image slicer. The pupil mirrors form the entrance slit of the spectrograph as well as a common pupil for the system, and the light from the pupil mirrors proceeds to the left again, reaching the collimator mirror at the upper right center in Figure 5. After the collimator the light is split into two beams (red and blue) by a dichroic. The optical paths of the instrument's red and blue channels are composed of the same elements. Each has a fold mirror, a transmission VPH grating, a spectrograph camera, and the detector. The grating is mounted on a rotation stage for angle adjustment. The camera and detector are mounted on a polar motion stage that can swing the camera and detector through a range of dispersion from the grating to allow selection of the desired bandpass and maximize efficiency for the desired bandpass. 
KCWI will have multiple gratings, and the preferred configuration is to provide an automated system for interchanging the gratings.

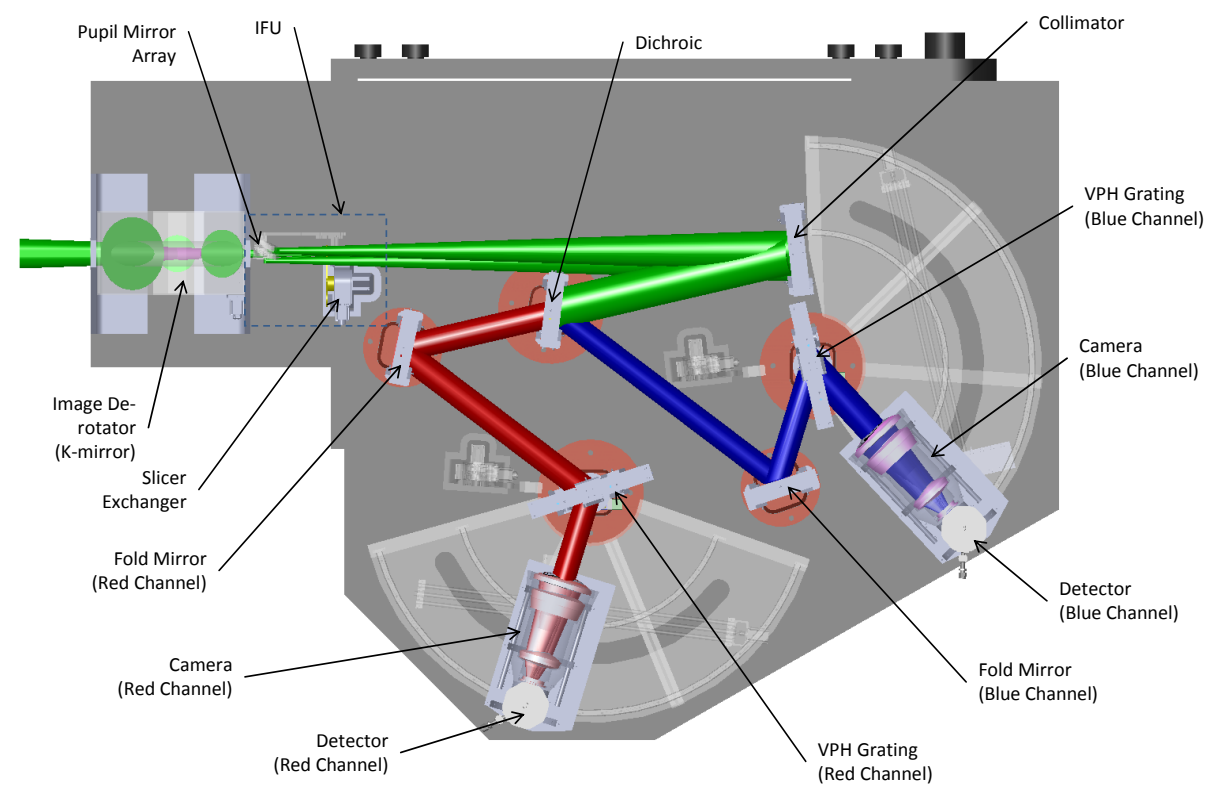

Figure 5: Plan view of the KCWI conceptual layout

The blue channel of KCWI will cover the wavelength range from 0.35 to $0.56 \mu \mathrm{m}$, and the red channel will cover the wavelength range from 0.53 to $1 \mu \mathrm{m}$. The candidate sampling scales for the KCWI IFU are $0.35 ", 0.7 "$ ", and 1.4 " slice widths x 24 slices, giving fields of view of 20" x 8.4", 20" x 16.8", and 20" x 33.6". The cameras will use detectors with $15 \mu \mathrm{m}$ pixels providing $\sim 4$ pixel sampling over a 0.6 " seeing disk in the spatial direction. The red camera will use a $4 \mathrm{k} \times 4 \mathrm{k}$ red optimized thick substrate CCD, and the blue camera will use an e2v technologies 231-84 4k x 4k $15 \mu \mathrm{m}$ pixel back illuminated, thinned CCD with a broad band AR coating. A selection of $\sim 12 \mathrm{VPH}$ gratings will allow spectral resolutions from 1,000 to 20,000 with excellent sensitivity. The total throughput including the telescope and atmosphere is predicted to be at least $20 \%$ over the full wavelength range $(0.35 \mu \mathrm{m}$ to $1 \mu \mathrm{m})$ with a mid-band peak of $35 \%$.

KCWI will also benefit from the heritage of previous WMKO instruments for its electronics and control software. Appropriate heritage from the recent LRIS red upgrade will be used for the red channel detector and read out system and the supervisory controls and motion control will be a combination of CWI heritage and WMKO standard electronics designs. Software will be based on the WMKO standard client-server architecture with DS9 used for image display. A custom data reduction pipeline for the IFS will be developed using IDL. The KCWI team and the Observatory hope to fast track the development of KCWI with delivery of KCWI to the Keck II telescope in late 2012.

\subsection{The Keck Next Generation Adaptive Optics Facility}

The Keck Next Generation Adaptive Optics (NGAO) facility ${ }^{[17]}$ is a new LGS AO system for the Keck II telescope that is intended to expand the range of science questions that can be addressed with LGS AO by providing near diffractionlimited performance in the near-IR (K band Strehl $\sim 80 \%$ ) over narrow fields $(<30$ " diameter) with modest correction down to $\sim 700 \mathrm{~nm}$, high sky coverage, improved sensitivity and contrast, and improved photometric and astrometric accuracy. NGAO is a collaboration among CIT, UCSC, and WMKO. Peter Wizinowich (WMKO) is the project manager, Sean Adkins (WMKO) is the science instrument lead, Richard Dekany (CIT) is leading the systems engineering activities and the design of the wavefront sensing systems, Don Gavel (UCSC) is leading the AO system and RTC design, Jason Chin (WMKO) is leading the laser guide star facility design, and Claire Max (UCSC) is the project scientist.

A schematic of the NGAO facility is shown in Figure 6. A multiple LGS facility using three 20 watt lasers and a center projection telescope located behind the telescope secondary produces a central fixed laser asterism consisting of 4 LGS beacons for tomographic measurement of the atmospheric turbulence (overcoming the cone effect) and three patrolling LGS beacons to sharpen three tip-tilt reference stars to overcome tip-tilt anisoplanatism and provide improved sky coverage by allowing the use of fainter tip-tilt stars. 
The AO system's optical path is contained within a cooled enclosure as shown in Figure 6. The optical path is cooled to approximately $-15{ }^{\circ} \mathrm{C}$ which keeps the thermal background contribution from the $\mathrm{AO}$ system in the near infrared at a level commensurate with the contribution of the telescope. After a k-mirror image de-rotator low order wavefront correction is performed by a low order AO relay with a FOV of 120 " using a conventional deformable mirror (DM) with 20 actuators across the telescope pupil on a $5 \mathrm{~mm}$ pitch. This provides closed loop correction of the LGS images, reducing the dynamic range demanded from the LGS wavefront sensors and also reducing the stroke requirements for a second high order DM that is used to correct the science field. A beam splitter sends the $589 \mathrm{~nm}$ light from the LGSs through a window in the wall of the cold enclosure to the object selection mechanism of the LGS wavefront sensor unit located outside the cold enclosure. This mechanism provides fixed pick-offs for the central 4 LGS asterism and three movable pick-offs to acquire the patrolling LGSs pointed at the tip-tilt stars.

After the low order DM the three pick-off arms are used to send the three tip-tilt star images to three low order wavefront sensors. Each low order wavefront sensor operates in the near-IR using an H-2RG detector and has open loop AO correction provided by a $32 \times 32$ MEMS DM. A selection mirror and field steering mirrors are also located after the low order DM to feed the Keck II arm of the Keck Interferometer through a second window in the cold enclosure. Also located after the first DM is the high order AO relay with a FOV of 40 " x 60 " that provides open loop correction for the science field using a 64 x 64 MEMS DM. After the high order AO relay a selectable dichroic beam splitter is provided to support a natural guide star mode, followed by a selection mirror that routes to science light to one of two science instrument ports.

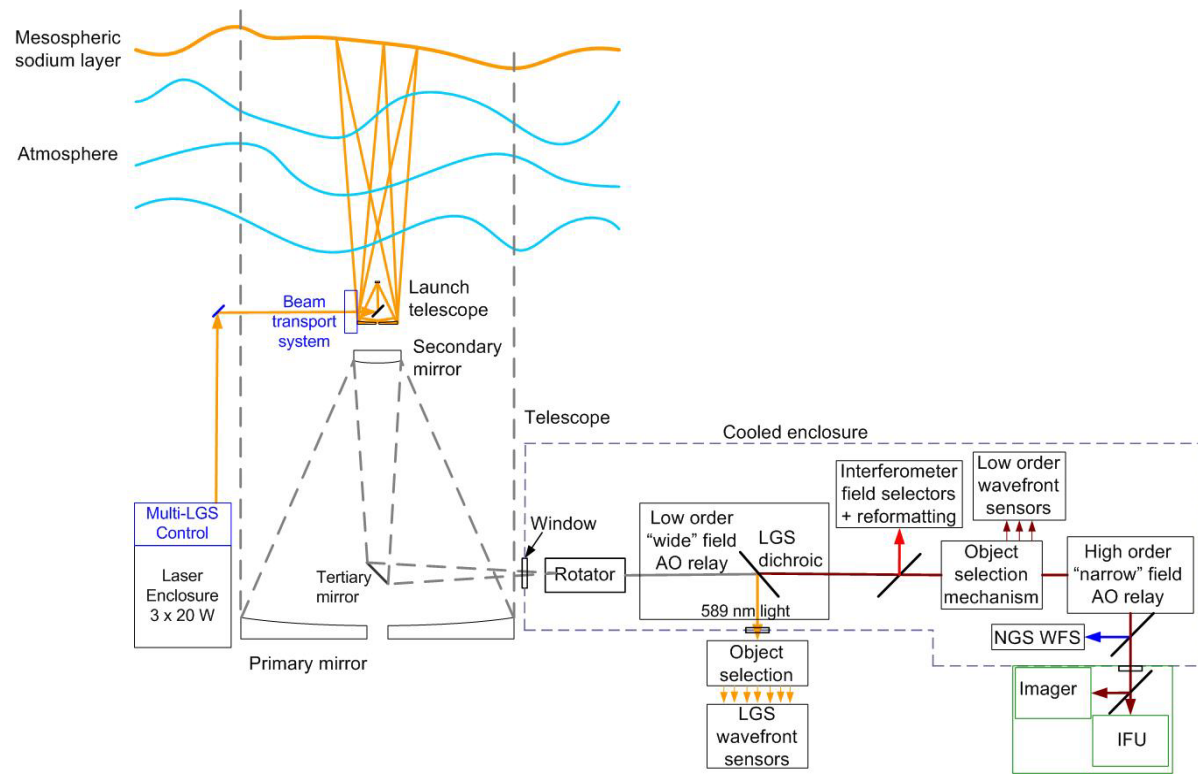

Figure 6: Schematic of the NGAO facility

Figure 7 shows a perspective view of the cooled AO enclosure with its roof removed. Starting at the left side of the figure, and continuing clock wise around the enclosure, the figure shows the LGS wavefront sensor assembly, the interferometer's dual star module, a place holder for a second science instrument, and finally the first light science instrument DAVINCI which provides both imaging and integral field spectroscopy from $0.7 \mu \mathrm{m}$ to $2.4 \mu \mathrm{m}$.

An example performance estimate for the NGAO system is shown in Figure 8. As the figure shows the sky coverage is excellent due to the near-IR corrected tip-tilt stars. Simulations also show that the system's performance is robust under varying conditions of seeing, laser return and zenith angle. This robust performance contributes to a more consistently beneficial level of AO correction for a wide range of science cases.

The NGAO system's preliminary design phase began in May 2008, and NGAO passed its preliminary design review in June 2010. The project is now waiting for funding and the approval to proceed with the detailed design phase. First NGAO science is expected in late 2015 based on the current project schedule. 


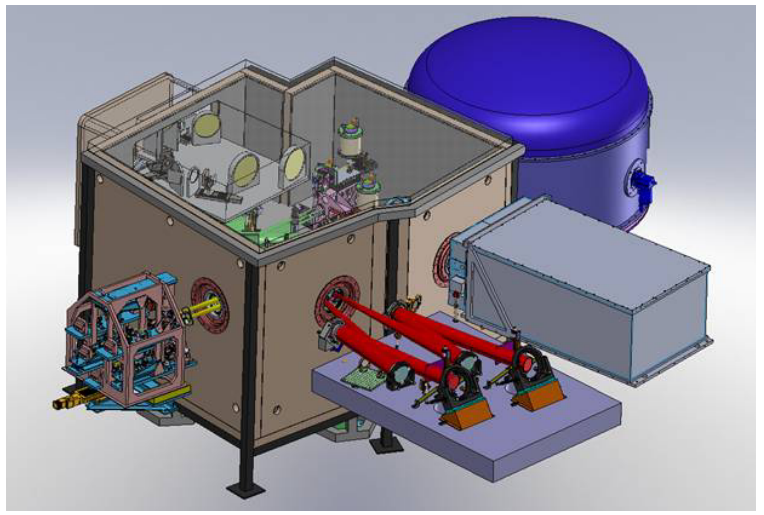

Figure 7: Perspective view of the NGAO system and science instruments at Nasmyth

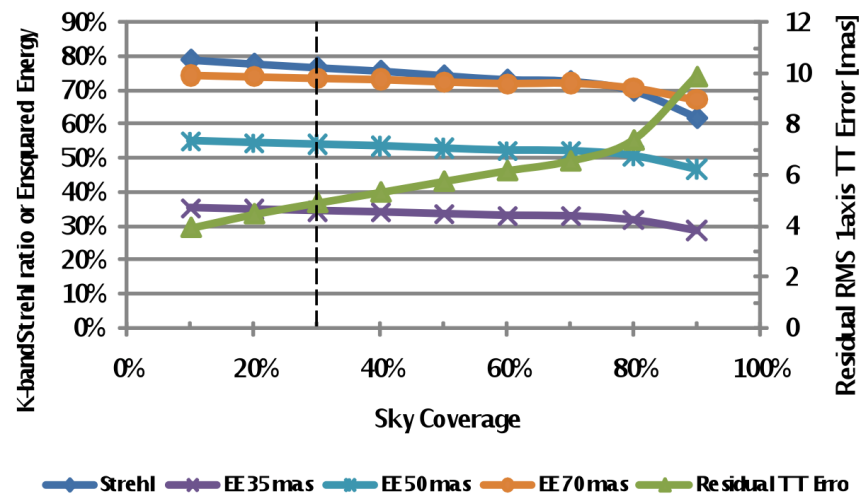

Figure 8: K-band Strehl, ensquared energy (within 35, 50 and 70 milli-arcseconds) and residual tip-tilt error versus sky coverage for the galaxy assembly science case $\left(30^{\circ}\right.$ zenith angle, $\geq 60^{\circ}$ galactic latitude, 30 minute integration)

\subsection{DAVINCI}

DAVINCI $^{[18]}$, the Diffraction limited Adaptive optics Visible and Infrared iNtegral field spectrograph and Coronagraphic Imager is the first light science instrument for NGAO. The instrument is being developed in collaboration with UCLA, UCSC, and UCO/Lick. DAVINCI successfully completed its preliminary design review as part of the NGAO preliminary design review in June 2010.

DAVINCI is a fully cryogenic instrument providing imaging and integral field spectroscopy at the diffraction limit over a wavelength range of $0.7 \mu \mathrm{m}$ to $2.4 \mu \mathrm{m}$. The imaging mode has a fixed plate scale of 7 milliarcseconds (mas) and the FOV is 28.7" x 28.7" using a Teledyne Hawaii-4RG detector with 4096 x 4096 pixels and a $2.5 \mu \mathrm{m}$ cut-off wavelength. The imager provides a selectable coronagraph mask and a large selection of photometric, continuum, and narrowband filters. A tracking cold pupil mask is provided for $\mathrm{H}$ and $\mathrm{K}$ band observations, and an additional selection of pupil masks is provided for the shorter wavelength bands and for the coronagraph mode. A pupil imaging mode is also provided for alignment of the instrument with the AO system. The central portion ( 6.5") of the DAVINCI FOV can be sent to the IFS, allowing simultaneous spectroscopy and imaging. The DAVINCI IFS mode provides with $112 \times 60$ spatial samples over a wavelength range of $0.7 \mu \mathrm{m}$ to $2.4 \mu \mathrm{m}$. Samplings scales of 10,35, and 50 mas are provided, resulting in FOVs of $1.12 "$ x $0.6 ", 3.92 "$ x 2.1", and 5.6" x 3". Fixed gratings are provided for each wavelength range, operating in the first order near the blaze angle with $\mathrm{R} \sim 4,000$. The IFS is optimized for narrow band observations ( $\sim 5 \%$ bandpass) and uses a lenslet image slicer combined with novel reformatting optics to provide 6 virtual slits $(680$ pixels per spectra) on a H-4RG detector with a $2.5 \mu \mathrm{m}$ cut-off wavelength. All of the reflective optics in DAVINCI are coated with bare gold.

Figure 9 shows the opto-mechanical layout of the instrument. Components are arranged on two levels, and the dewar is cylindrical with an outer diameter of $\sim 2 \mathrm{~m}$. The $120 \mathrm{~mm}$ diameter dewar entrance window is coupled by an insulated bellows to the cooled enclosure of the AO system, eliminating the need to provide a heating system for the window. The coronagraph mask wheel is located at the AO system focus inside the dewar and provides a selection of occulting spots, and a field stop for the pupil imaging mode. The next section of the instrument consists of an OAP pair and the filter wheels and pupil mask wheel, all of which are shared by the imager and IFS modes. The first OAP in DAVINCI is matched to the second OAP in the high order relay of the AO system, and this approach provides a higher quality pupil image at the DAVINCI pupil mask. The pupil image is $25 \mathrm{~mm}$ in diameter and the filters are $40 \mathrm{~mm}$ in diameter, with a total of 45 filters provided on three wheels ( 15 per wheel plus an open position) located in the collimated space near the pupil. The second OAP forms an intermediate focus, and near this focus a mirror is located that has a hole to allow light to pass to the IFS while allowing the rest of the field to pass on to the imager. The mirror can be rotated about its center which allows the hole to be moved out of the beam, sending the entire FOV to the imager.

The imager consists of a second OAP pair and a $\mathrm{BaF}_{2}$ field flattener. A wheel mechanism is also provided to insert a lens for pupil imaging. This pupil imager is optimized for $\mathrm{K}$ band. The detector head is based on the detector head developed 
for MOSFIRE, but adapted to the H-4RG. A focus adjustment will be provided for initial set-up of the imager optical path when cold.

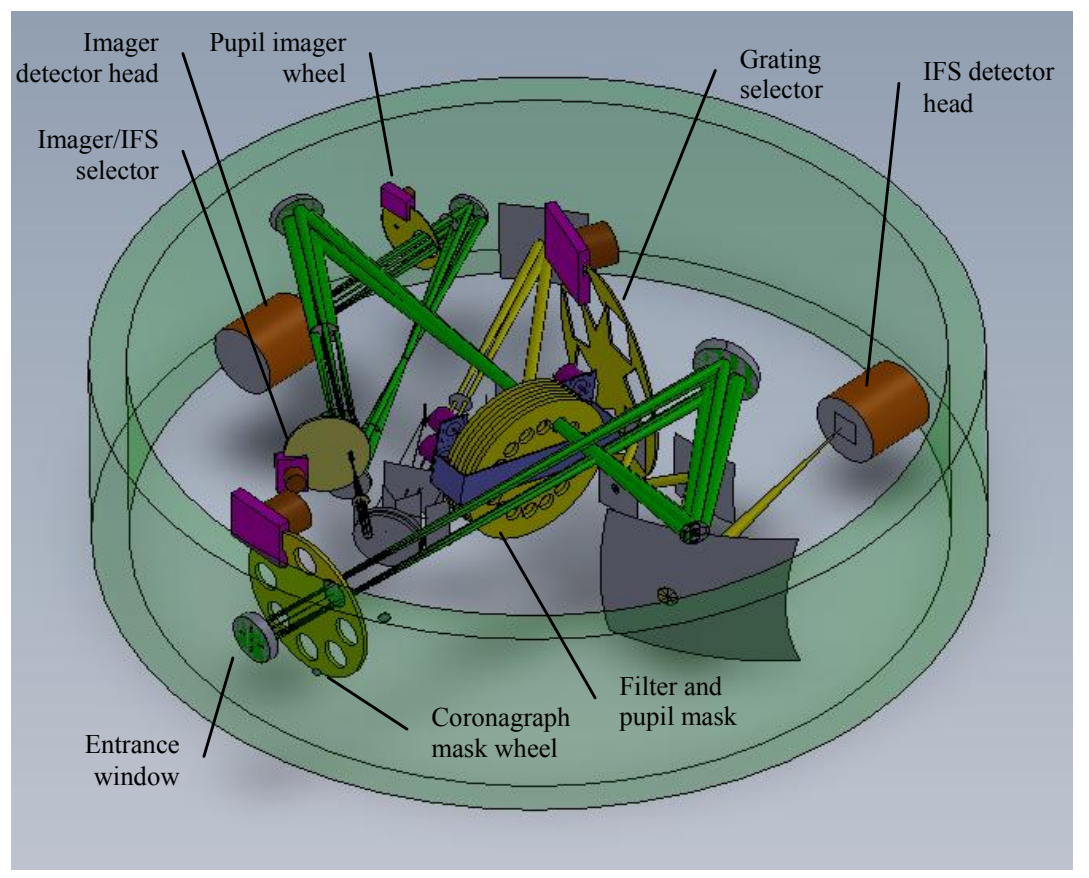

Figure 9: DAVINCI's opto-mechanical layout

After the IFS/imager selection mirror a scale changer with three selectable scales images the central portion of the intermediate focus onto the IFS lenslet array which consists of $112 \times 60$ lenslets on a $250 \mu \mathrm{m}$ pitch. This is followed by the reformatting system used to produce the virtual slits. This consists of a field magnifier to enlarge the array of pupil images from the lenslet and provide the space needed to perform the reformatting. A tent mirror splits the field into two halves on the long axis, and each half is then sent to the reformatting mirrors. The reformatting mirrors are arranged so that each half of the field is effectively split into two halves on the short axis, resulting in 4 quadrants of $56 \times 30$ lenslets as illustrated in Figure 10.

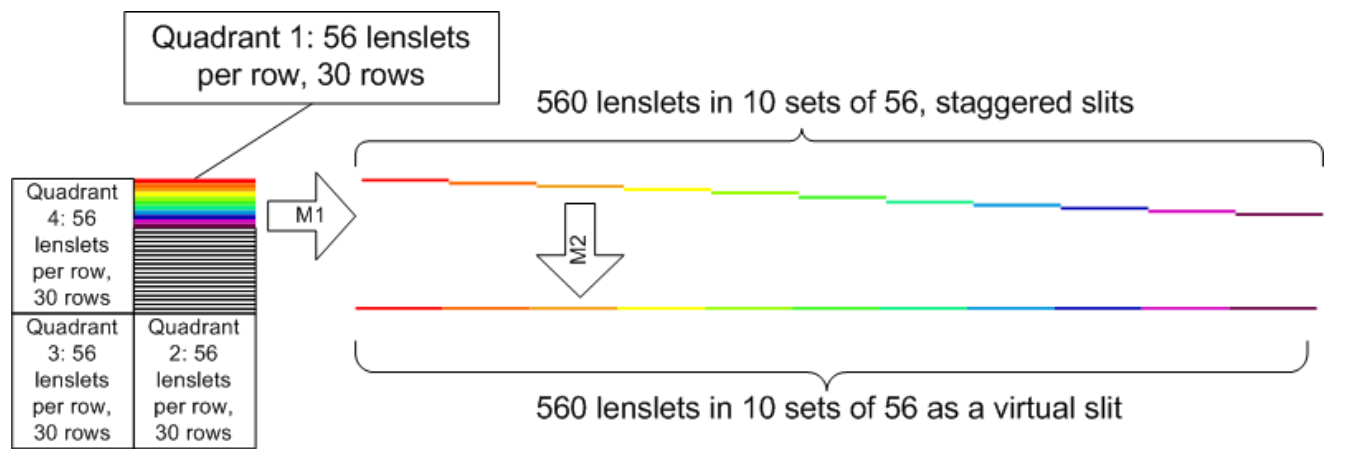

Figure 10: IFS virtual slit reformatting process

The first mirror is an array of spherical mirrors similar to a conventional mirror slicer, and this reformats the rows of pupil images from the lenslet into three staggered lines consisting of 10 sections of 56 lenslets in each staggered line. The second reformatting mirror consists of 10 spherical mirrors that align each of the 10 sections to form a virtual slit of 560 pupil images. By repeating this process for the other 3 quadrants we obtain six virtual slits each consisting of 1120 pupil images.

The six virtual slits are then collimated by a single aspheric collimating mirror followed by a grating wheel carrying 7 gratings ( 1 for each wavelength band, I, Z, Y, J, and H, and 2 for $\mathrm{K}$ band) and a plane mirror. A three mirror anastigmat (TMA) forms the camera and this is followed by a second detector head using an H-4RG detector. The DAVINCI TMA 
design is based on the IRIS TMA design by Brian Bauman ${ }^{[19]}$. Work is ready to start on the detailed design phase of DAVINCI in conjunction with the next phase of the overall NGAO facility design.

\section{CONCLUSIONS}

As we have shown through this review of recently completed developments and developments in progress, the community of observers at WMKO continues to benefit from the strong collaborations between the Observatory and the instrumentation development teams at CIT, UCLA, UCO/Lick and UCSC. MOSFIRE, KCWI, and the NGAO facility and instrumentation offer leading capabilities in both seeing limited and adaptive optics instrumentation that is essential to enabling our community's scientific leadership in the next decade.

\section{ACKNOWLEDGEMENTS}

The W. M. Keck Observatory is operated as a scientific partnership among the California Institute of Technology, the University of California, and the National Aeronautics and Space Administration. The Observatory was made possible by the generous financial support of the W. M. Keck Foundation. The Observatory would like to acknowledge Gordon and Betty Moore for their support of the MOSFIRE instrument. This material is based in part upon work supported by AURA through the National Science Foundation under Scientific Program Order No. 5 as issued for support of the Telescope Systems Instrumentation Program (TSIP), in accordance with Proposal No. AST-0335461 submitted by AURA. This material is based in part upon work supported by the National Aeronautics and Space Administration under Grant and Cooperative agreement No. NNX08AH49A. This material is also based in part upon work supported by the National Science Foundation under Cooperative Agreement No. AST-0084699.

\section{REFERENCES}

[1] Ragland, S. D., Akeson, R. L., Colavita, M. M., Millan-Gabet, R., Julien M. Woillez, J. M., Wizinowich, P. L., Appleby, E., Berkey, B. C., Cooper, A., Dahl, W., Felizardo, C., Herstein, J., Hrynevych, M. A., Medeiros, D. W., Morrison, D., Panteleeva, T., Pott, J., Smith, B., Summers, K. R., Tsubota, K., Tyau, C., \& Wetherell, E. "Recent progress at the Keck interferometer [Invited Paper]", In Proceedings of the SPIE 7734, 01. (2010).

[2] Woillez, J. M., Colavita, M. M., Eisner, J. A., Ghez, A. M., Hillenbrand, L., Millan-Gabet, R., Monnier, J. D., Pott, J., Ragland, S. D., \& Wizinowich, P. L. "ASTRA: The astrometric and phase-referencing astronomy upgrade for the Keck interferometer", In Proceedings of the SPIE 7734, 37. (2010).

[3] McLean, I. S., \& Adkins, S. M. "Instrumentation at the W. M. Keck Observatory [invited paper]", In Proceedings of the SPIE 7014, 701402. (2008).

[4] Eisenstein, D., Hammel, H., Hillenbrand, L., Johns-Krull, C., Koo, D., Levenson, N., Matheson, T., McWilliam, A., Monnier, J., Najita, J., Prato, L., Ramsey, L., Redfield, S., \& Soifer, T. "Final Report of the Committee on Access to Large Telescopes for Astronomical Instruction and Research (ALTAIR)", (2009). Retrieved from www.noao.edu/system/altair/files/ALTAIR_Report_Final.pdf

[5] Liu, M. C. "LGS AO science impact: present and future perspectives", In Proceedings of the SPIE 7015, 701508701508-13. (2008).

[6] Ragland, S., Wizinowich, P., Akeson, R., Colavita, M., Appleby, E., Berkey, B., Booth, A., Cooper, A., Crawford, S., Dahl, W., Felizardo, C., Garcia-Gathright, J., Gathright, J., Herstein, J., Hrynevych, M., Koresko, C., Ligon, R., Medeiros, D., Mennesson, B., Millan-Gabet, R., Morrison, D., Paine, C., Parvin, B., Panteleeva, T., Serabyn, E., Smith, B., Summers, K., Tsubota, K., Tyau, C., Wetherell, E., \& Woillez, J. "Recent progress at the Keck Interferometer: operations and $\mathrm{V}^{2}$ science", In Proceedings of the SPIE 7013, 70130B-70130B-12. (2008).

[7] Oke, J. B., Cohen, J. G., Carr, M., Cromer, J., Dingizian, A., Harris, F. H., Labrecque, S., Lucinio, R., Schaal, W., Epps, H., \& Miller, J. “The Keck low-resolution imaging spectrometer", In Publications of the Astronomical Society of the Pacific 107, 375-385. (1995).

[8] Bebek, C. J., Groom, D. E., Holland, S. E., Karcher, A., Kolbe, W. F., Palaio, N. P., Roe, N. A., Turko, B. T., \& Wang, G. "Development of fully depleted back-illuminated charge-coupled devices", In Proceedings of the SPIE 5499, 140-150. (2004). 
[9] Rockosi, C. M., Stover, R., Kibrick, R. I., Lockwood, C., Peck, M., Cowley, D. J., Bolte, M., Adkins, S. M., Alcott, B., Allen, S. L., Brown, W. F., Cabak, G. F., Deich, W. T. S., Hilyard, D. F., Kassis, M. F., Lanclos, K., Lewis, J. P., Pfister, T., Phillips, A. C., Robinson, L., Saylor, M., Thompson, M., Ward, J., Wei, M., \& Wright, C. A. "The low-resolution imaging spectrograph red channel CCD upgrade: Fully depleted, high-resistivity CCDs for Keck", In Proceedings of the SPIE 7735, 27. (2010).

[10] Adkins, S. M., Cohen, J., Aycock, J., Bell, J., Cohen, R., Cooper, A., Goodrich, B., Johnson, J., Kwok, S. H., Lyke, J., Neyman, C., Nordin, T., Panteleev, S., Tolleth, G., \& Tsubota, M. "MAGIQ at the W. M. Keck Observatory, initial deployment of a new acquisition, guiding and image quality monitoring system", In Proceedings of the SPIE 7014, 70141U. (2008).

[11] Kwok, S. H., Johnson, J., Adkins, S. M., \& McCann, K. "The software for MAGIQ: a new acquisition, guiding and image quality monitoring system at the W. M. Keck Observatory", In Proceedings of the SPIE, 7019, 10. (2008).

${ }^{[12]}$ Kwok, S. H., \& A. Conrad. "Mira: a case study", In Proceedings of the SPIE 4848, 414-424. (2002).

[13] Sawruk, N. W., ,Lee, I., Jalali, M. P., Prezkuta, Z., Groff, K. W., Roush, J., Rogers, N., Tiemann, B., Hannon, S. M., Alford, W. J., d'Orgeville, C., Fesquet, V., Oram, R., Adkins, S. M., \& Grace, K. "System overview of 30 W and 60 W 589 nm guidestar laser systems", In Proceedings of the SPIE 7763, 69. (2010).

[14] Chin, J. Y. C., \& Stalcup, T. "Keck I laser guide star AO system integration”, In Proceedings of the SPIE $7736,66$. (2010).

[15] McLean, I. S., Steidel, C. C., Epps, H. W., Matthews, K. Y., \& Adkins, S. M. "Design and development of MOSFIRE: the multi-object spectrometer for infrared exploration at the Keck Observatory", In Proceedings of the SPIE 7735, 49. (2010).

[16] Martin, D. C., Moore, A. M., Adkins, S. M., Chang, D. Y., Delacroix, A., Epps, H. W., Matuszewski, M., Morrissey, P., \& Rahman, S. "The Keck cosmic web imager", In Proceedings of the SPIE 7735, 22. (2010).

[17] Wizinowich, P. L., Adkins, S. M., Bell, J. M., Chin, J. Y. C., Stalcup, Jr., T. E., Johansson, E. M., Johnson, J. M., Medeiros, D. W., Morrison, D., Neyman, C. R., Panteleev, S., Pollard, M., Wetherell, E., Gavel, D. T., Max, C. E., Kupke, R., Reinig, M. R., McGrath, E. J., Lockwood, C., Dekany, R. G., Bouchez, A. H., Velur, V. N., \& Marchis, F. "W. M. Keck Observatory's next generation adaptive optics facility", In Proceedings of the SPIE $7736,19$. (2010).

[18] Adkins, S. M., Bell, J. M., Conrad, A., Fitzgerald, M., Kupke, R., Larkin, J. E., Laiterman, L., Lyke, J., Max, C. E., McGrath, E. J., Pollard, M., Panteleev, S., Thomas, S. J., \& Wizinowich, P. "DAVINCI: A high-performance imager and integral field spectrograph for the W. M. Keck Observatory's next generation adaptive optics facility”, In Proceedings of the SPIE 7735, 287. (2010).

[19] Moore, A. M., Bauman, B. J., Barton, E. J., Crampton, D., Delacroix, A., Larkin, J. E., Simard, L., \& Wright, S. A. "The infrared imaging spectrograph (IRIS) for TMT: Spectrograph design", In Proceedings of the SPIE $7735,87$. (2010). 